\title{
ESDA2012-82725
}

\section{MODELLING THE HEAT DURING THE INJECTION STRETCH BLOWING MOULDING: INFRARED HEATING AND BLOWING MODELLING}

\author{
Yun Mei Luo, Luc Chevalier and Françoise Utheza \\ Université Paris-Est, Laboratoire Modélisation et Simulation Multi Echelle, MSME UMR 8208 CNRS, 5 bd \\ Descartes, 77545 Marne-la-Vallée, France
}

\section{KEYWORDS}

Initial heating conditions, thermal properties, thermo-viscohyperelastic model

\begin{abstract}
Effects of temperature: initial heating conditions or self heating during the process, are very important during the injection stretch blow moulding (ISBM) process of PET bottles. The mechanical characteristics of the final products, which are mainly controlled by the final thickness and the orientation of the molecular chains, depend strongly on the process temperature. Modelling the heat transfer during the ISBM process is therefore necessary. In the first part of this paper, an experimental study is presented in order to measure the initial temperature distribution and to identify the thermal properties of the PET. An infrared camera has been used to determine the surface temperature distribution of the PET sheets which are heated by infrared (IR) lamps. The Monte Carlo method is used to identify the parameters best fit from the temperature evolution. In the second part, a thermo-viscohyperelastic model is used to predict the PET behaviour, taking into account the strain rate and temperature dependence. A finite element approach implemented in matlab is used to achieve the numerical simulation.
\end{abstract}

\section{INTRODUCTION}

The effects of temperature, initial heating conditions or self heating during the process, are of fundamental importance during the injection stretch blow moulding (ISBM) process of PET bottles. They control the preform temperature distribution and strongly affect the blowing kinematics via the important influence of temperature on mechanical behaviour. The temperature affects the orientation induced by biaxial stretching, which in turn, affects mechanical properties of PET. Besides, the final thickness distribution is controlled by the temperature distribution inside the preform. Therefore, thermal properties are one of the most important variables in the ISBM.

In order to achieve accurate simulation of the ISBM process, it is necessary to: (i) measure the initial temperature distribution of the preform when the blowing operation begins; (ii) follow the history of the temperature field and consequently to identify the thermal properties of the PET; (iii) to model the behaviour law of PET coupled to the thermal laws.

In this work, we modelled the PET behaviour in ISBM strain rate and temperature conditions by a thermoviscohyperelastic model which has been inspired from Figiel and Buckley 2009 [1] and already presented by Luo et al. 2011 [2].

A procedure is proposed for the identification of the thermal parameters from experimental results of a test where PET sheets are heated using infrared (IR) lamps. The Monte Carlo method is used to provide the parameters best fit from the temperature evolution measured on the face in front of the lamps and from the rear face. The beginning of the temperature evolution gives information on the IR flux and the final stage of the heating gives information on the convection exchange. Difference between the front and the rear faces gives information on the PET conductivity.

Using a finite element approach implemented in Matlab, the coupled thermo-visco-hyperelastic model has been used to simulate the ISBM process. We implement an axi-symmetric version of the visco-hyperelastic model coupled to temperature for the numerical simulation. The self heating effect is not negligible and has an important effect on the viscohyperelastic 
model. Therefore we carried out non-isothermal viscohyperelastic simulations in order to compare with the experimental data [4].

\section{EXPERIMENTAL PROCEDURE}

According to Figure 1, the experimental apparatus which consisted in measuring, by thermal imaging, a PET sheet heating by infrared lamps. An FLIR B250 infrared camera with the wavelength range $7.5-13 \mu \mathrm{m}$ is used to evaluate the surface temperature distribution. Contrary to the thermocouples which are easy to use but gives a local measure of the temperature, the thermal imaging allows the determination of the surface temperature. The surface dimension of the sheet is $60 \mathrm{~mm} \times 125 \mathrm{~mm}$ with the thickness $1 \mathrm{~mm}$.

We tested three different distances between the lamps and the PET sheet: $6.5 \mathrm{~cm}, 13 \mathrm{~cm}$ and $19.5 \mathrm{~cm}$. For a constant heating IR flux, we can notice that the temperature decreases while this distance increases. This a logical result because the intensity of the radiation decreases in proportion to the inverse of the square of the distance [5] which can be confirmed by plotting the radiation flux vs the distance. Increasing the distance, the air convection process also takes more importance and in regard of radiation.

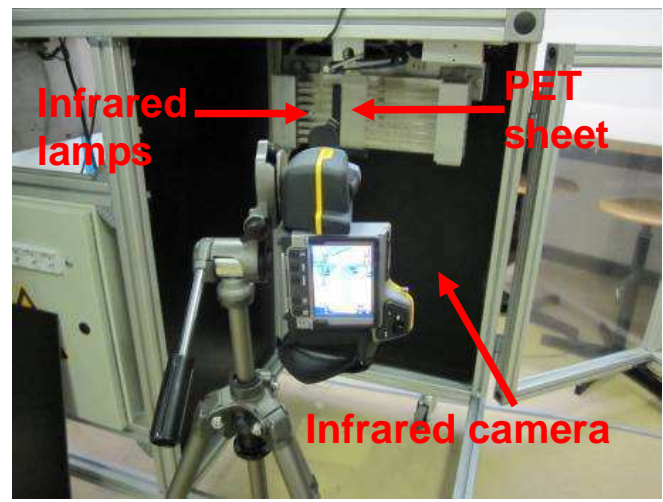

FIGURE 1. Experimental heating set-up
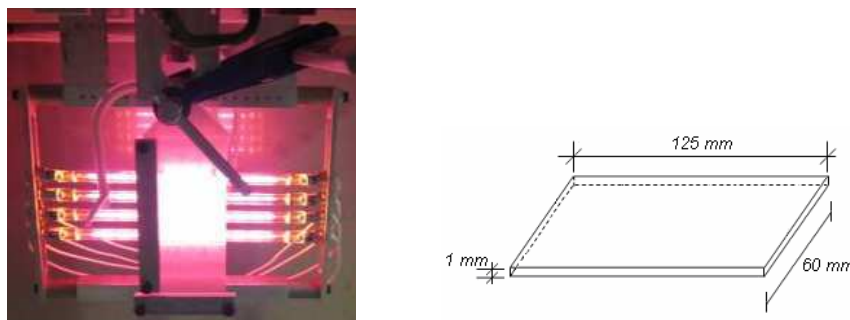

(a)

FIGURE 2. (a) IR lamps with PET sheet; (b) the dimension of the PET sheet

\section{IDENTIFICATION OF THE THERMAL PROPERTIES}

The software FLIR quick report is used to measure accurately the temperature. The thickness of the PET sheet is $1 \mathrm{~mm}$. So the PET may be considered like an opaque medium which means that only the radiation emitted from the sheet surface is intercepted by the camera's sensor [6,7]. The black paint used is assumed to be opaque but because we are not able to quantify its emissivity identification will be managed from thermocouple measures. Nevertheless, this temperature field visualization shows that some temperature heterogeneities appear on the edges of the sheet surface. According to these results, we can assume that the temperature is homogeneous in the plane of the sheet and only varies in the thickness direction. Therefore, the identification can be done form a one dimensional model.

The temperature balance equation in the $1 \mathrm{D}$ case can be written:

$$
\rho C_{p} \dot{T}-k \frac{\partial^{2} T}{\partial z^{2}}=0
$$

where: $\rho$ the mass density, $C_{p}$ the specific heat capacity and k is the material's conductivity.

With the boundary conditions:

$k \underline{\nabla} T . \underline{n}=\varphi_{s}-h_{f}\left(T-T_{\infty}\right) \quad$ on the face in front of the lamps;

$$
k \underline{\nabla} T . \underline{n}=h_{r}\left(T-T_{\infty}\right) \quad \text { on the rear face. }
$$

where $\varphi_{s}$ is the heat flux resulting from IR lamps. The convective heat transfer coefficient $h$ is different as the face in front of the lamps or the face in the back. $T_{\infty}$ is the surrounding bulk temperature.

While the IR flux $\varphi_{s}$ estimation can be obtained at the beginning of the temperature evolution, the convection exchange $h$ estimation can be rather got from the final stage of the heating. The PET conductivity $k$ can be calculated from the difference between the front and the rear faces.

The density $\rho$ is measured directly and the Monte Carlo method is used to identify the other parameters to best fit the experimental results. So the thermal proprieties are listed in the table1. This identification shows that $h_{f}$ is almost useless in regard of the rear coefficient $h_{r}$.

TABLE 1. THE VALUE OF THERMAL PROPERTIES

\begin{tabular}{c|c}
\hline Parameters & Values \\
\hline$\rho\left(\mathrm{kg} / \mathrm{m}^{3}\right)$ & 1345 \\
\hline$C_{p}(\mathrm{~J} / \mathrm{kg} . \mathrm{K})$ & 1880 \\
\hline$k(\mathrm{~W} / \mathrm{m} . \mathrm{K})$ & 0.102 \\
\hline$h_{f}\left(\mathrm{~W} / \mathrm{m}^{2} . \mathrm{K}\right)$ & 0 \\
\hline$h_{r}\left(\mathrm{~W} / \mathrm{m}^{2} \cdot \mathrm{K}\right)$ & 28.59 \\
\hline$\varphi s\left(\mathrm{~W} / \mathrm{m}^{2}\right)$ & 1460 \\
\hline
\end{tabular}


Figure 3 represents the experimental temperature evolution on the surface in front of the lamps $T_{f}$ and the one on the rear face $T_{r}$. With the identified parameters, the curves obtained fit the experimental results (points).

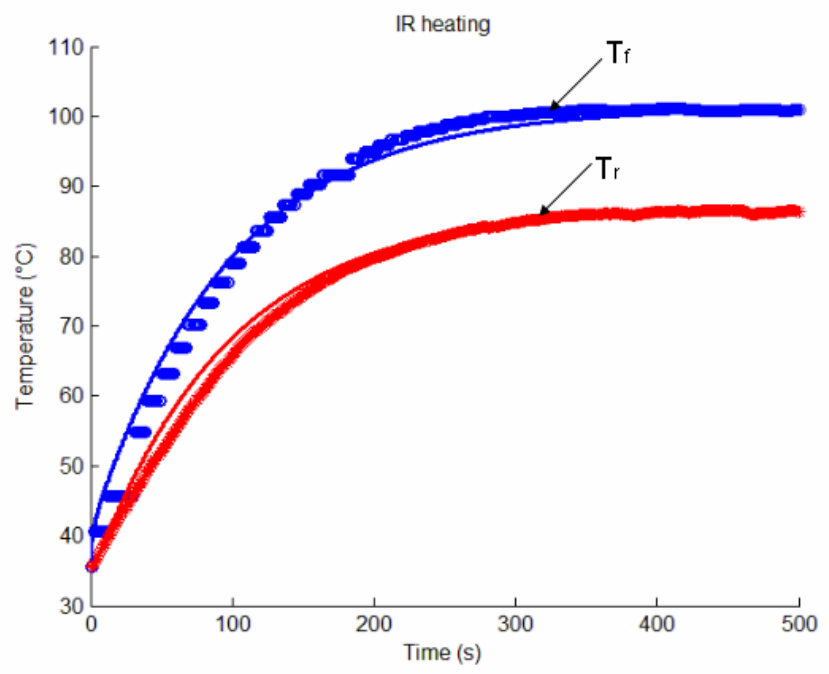

FIGURE 3. The experimental results (points) and the numerical results (curves) with optimal thermal properties

\section{THE VISCOHYPERELASTIC BEHAVIOUR OF PET}

We proposed an incompressible visco-hyperelastic model with nonlinear forms for both elastic and viscous parts [2].

$$
\begin{aligned}
& \underline{\underline{\sigma}}=2 \eta_{N} \underline{\underline{D}}+\underline{\underline{\hat{\sigma}}}-p \underline{\underline{I}}-q \underline{\underline{I}} \\
& \left\{\begin{array}{l}
\hat{\hat{\sigma}}=2 G\left(\varepsilon_{e}\right) \underline{\underline{\hat{\varepsilon}_{e}}} \\
\hat{\underline{\hat{\sigma}}}=2 \eta\left(\varepsilon_{v}, \underline{\overline{\dot{\varepsilon}_{v}}}, T\right) \underline{\underline{D_{v}}}
\end{array}\right.
\end{aligned}
$$

where $\underline{\underline{\sigma}}$ is the Cauchy stress tensor, $\underline{\underline{D}}_{\underline{v}}$ is the symmetric part of the viscous velocity gradient, the subscript "^" denotes the deviatoric part of the tensor, $\eta_{N}$ is the small value of the viscosity of the Newtonian branch of the Zener like model used in order to solve the ill-conditioned problem. $\underline{\underline{\varepsilon}}_{\underline{e}}$ is the elastic part of the Eulerian strain measure defined by:

$$
\left.\underline{\underline{\varepsilon_{e}}}=\frac{1}{2} \underline{\underline{\left(B_{e}\right.}}-\underline{\underline{I}}\right)
$$

where $\underline{\underline{B}}_{e}$ is the elastic left Cauchy Green tensor. $p$ is a Lagrange multiplier associated to the global incompressibility condition, and $q$ is the multiplier associated to the incompressibility of the elastic part.

$$
\left\{\begin{array}{l}
\operatorname{det} \underline{\underline{B_{e}}}=1, \\
\operatorname{div} \overrightarrow{\vec{V}}=\operatorname{trace} \underline{\underline{D}}=0, \\
\operatorname{div} \vec{V}_{v}=\operatorname{trace} \underline{\underline{D_{v}}}=0
\end{array}\right.
$$

The assumption of an additive decomposition of elastic and viscous velocity gradient is adopted to describe the kinematic structure of this model:

$$
\underline{\underline{D}}=\underline{\underline{D_{e}}}+\underline{\underline{D_{v}}}
$$

Combining equations, 1,2 and 4 in the Oldroyd derivation of the elastic left Cauchy-Green tensor leads to:

$$
\frac{\delta \underline{B_{e}}}{\overline{\delta t}}+\frac{G}{\eta \underline{\underline{B_{e}}}} \underline{\underline{\underline{B_{e}}}}=0
$$

$G$ is the elastic shear modulus and $\eta$ is the viscosity. In order to represent the strain hardening and strain rate effect and temperature dependency, we choose two rheological functions for elastic and viscous parts: $G\left(\varepsilon_{e}\right)$ and $\eta\left(\varepsilon_{v}, \overline{\dot{\varepsilon}_{v}}, \mathrm{~T}\right) . \varepsilon_{e}$ is the equivalent elastic strain and $\overline{\dot{\varepsilon}_{v}}$ is the equivalent viscous strain rate.

We consider a Hart-Smith like model to represent the elastic part $G\left(\varepsilon_{e}\right)$ :

$$
G=G_{0} \exp \left(\Lambda\left(I_{1}-3\right)^{2}\right), I_{1}=\operatorname{trace}\left(B_{e}\right)
$$

For the non-linear viscous part of the model, we follow the same method as in Cosson and Chevalier [3] to represent macroscopically the strain hardening effect:

$$
\eta=\eta_{0}(T) h\left(\overline{\varepsilon_{v}}, T\right) \cdot f\left(\overline{\dot{\varepsilon}_{v}}\right)
$$

with $f\left(\overline{\dot{\varepsilon}}_{v}\right)=\left(1+\left(\lambda\left(\overline{\dot{\varepsilon}}_{v} / \dot{\varepsilon}_{r e f}\right)\right)^{a}\right)^{\frac{m-1}{a}}$

where $\dot{\mathcal{E}}_{\text {ref }}$ is a reference strain rate that can be taken equal to 1 $\mathrm{s}^{-1}$. The strain hardening effect is related to the $h$ function which increases continuously with $\bar{\varepsilon}_{v}$. We detailed the identification procedure for the $h$ function in [2]. The strain hardening effect is influenced by the temperature. Therefore, $h$ is a function of $T$ also:

$$
\eta_{0}(T) h\left(\overline{\varepsilon_{v}}, T\right)=\frac{\eta_{0}(T) \cdot\left(1-\exp \left(-K \overline{\varepsilon_{v}}\right)\right)}{\left(1-\overline{\varepsilon_{v}} / \varepsilon_{v \lim }(T)\right)^{N}}
$$

We choose the Williams-Landel-Ferry (WLF) model for the evolution of $\eta_{0}(T)$ :

$$
\ln \left(a_{T}\right)=\frac{-C_{1}\left(T-T_{r e f}\right)}{C_{2}+T-T_{r e f}}
$$

where $C_{1}$ and $C_{2}$ are the WLF parameters, $T_{r e f}=90^{\circ} \mathrm{C}$. We propose the evolution of $\varepsilon_{\text {vlim }}(T)$ in the following way:

$$
\varepsilon_{v \lim }=\varepsilon_{v \lim _{-} r e f}\left(1+\frac{B_{1}\left(T_{r e f}-T\right)}{\left(T-B_{2}\right)}\right)
$$

where $\varepsilon_{v \text { lim_ref }_{-}}=\varepsilon_{v \text { lim_ }_{-} 90^{\circ} \mathrm{C}}$.

During the stretching, the self-heating effect can be important in the specimen, especially for the high strain rate 
$\left(32 \mathrm{~s}^{-1}\right.$ for example). The adiabatic heating is due to the viscous dissipation which generates an increase of temperature in the PET sheet. We can estimate this temperature rise, for the case $32 \mathrm{~s}^{-1}$ at $90^{\circ} \mathrm{C}$, if assuming that the stretching is fast enough so that all dissipation is converted in self-heating (no exchange with ambient air). Consequently, the global temperature balance equation, which is integrated over the volume and over the time, writes:

$$
\int_{\Delta t} \int_{\Omega} \rho C_{p} \dot{T} d \Omega d t=\int_{\Delta t} \int_{\Omega} \underline{\underline{\sigma}}: \underline{\underline{D}} d \Omega d t
$$

The conduction term is neglected in this analytical solution because the biaxial stretching process is considered fast enough in regard of the time needed to propagate the temperature through the thickness. The characteristic time for diffusion is $t_{d}=\rho c_{p} e^{2} / k=57 \mathrm{~s}$ and the characteristic time for capacity is $t_{c}=\rho c e / h=132 s$. The time for the process is about $2 \mathrm{~s}$. If Eq.12 is used to estimate temperature as a post processing of the isothermal stretching simulation,, we can estimate the temperature has increased about $4^{\circ} \mathrm{C}$ when the stretch ratio reaches 2.8 . If we take this temperature evolution into account in our model of viscosity, the final value of the viscosity reduces of $25 \%\left(3.6 \times 10^{5}\right.$ Pa.s VS $4.8 \times 10^{5}$ Pa.s $)$ which shows that the self heating has a important effect on the PET behaviour. Therefore, a coupled themo-viscohyperelastique simulation of the biaxial elongation tests must be used to adjust the parameters of the thermo viscohyperelastic model used to represent the PET behaviour.

\section{NUMERICAL IMPLEMENTATION OF THE MODEL}

Considering the specific geometry of a PET perform, we implement an axi-symmetric version of the visco-hyperelastic model coupled to temperature in order to simulate accurately the ISBM process. Figure 4 shows the boundary conditions and the load applied to the circular sheet discretized by rectangular elements. The circular sheet is submitted to a radial velocity $V$ on radius $L$ and this case represents the equi-biaixal tension case: $\sigma_{r r}=\sigma_{\theta \theta}$. In order to compare the experimental results, the radius of the cylinder is $38 \mathrm{~mm}$ and the thickness is $0.75 \mathrm{~mm}$, which is representative of the PET specimen size $(76 \mathrm{~mm} \times 76 \mathrm{~mm} \times 1.5 \mathrm{~mm})$ of the test.

The related elongation rate $\dot{\lambda}$ can be given as the derivative of stretch ratio $\lambda$ with respect to time $t$. So the strain rate is: $\dot{\varepsilon}=\dot{\lambda} / \lambda$.

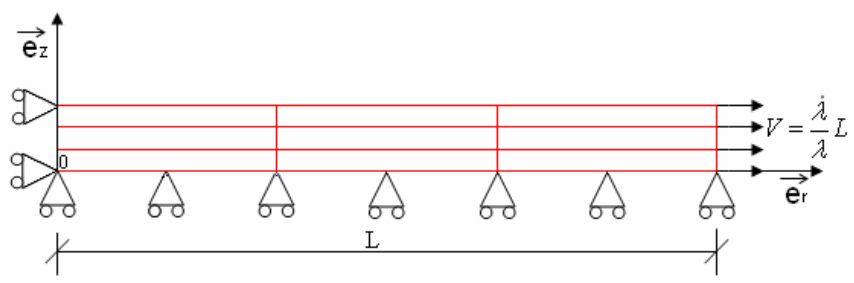

FIGURE 4. The cylinder under the radial velocity

The viscohyperelastic model is implemented in the Matlab environment using a finite element approach. An axi- symmetric formulation involving 4 fields (global velocity $V$, Lagrange multiplier $p$ associated with the global incompressibility condition, and multiplier $q$ associated with the incompressibility of the elastic part, the elastic left Cauchy Green tensor $B$ ) has been performed using rectangular elements. Some standard manipulations of equations 2, 4 and 6 lead to the weak form in the following 4 equations:

$$
\begin{aligned}
& \int_{\Omega} \underline{\underline{D}}^{*}: G \underline{\underline{\hat{B}_{e}}} d \Omega+\int_{\Omega} \underline{\underline{D^{*}}}: 2 \eta_{n} \underline{\underline{D}} d \Omega \\
& -\int_{\Omega} \underline{D}^{*}: \underline{I}\left(p_{e}+q\right) d \Omega-\int_{\partial \Omega_{F}} \underline{V}^{*} \underline{F}^{d} d S=0 \\
& \int_{\Omega} p^{*} \underline{\underline{I}}: \underline{\underline{D}} d \Omega=0 \\
& \int_{\Omega} q^{*}\left(\operatorname{det}\left(\underline{\underline{B_{e}}}\right)-1\right) d \Omega=0 \\
& \int_{\Omega} \underline{\underline{B_{e}}}{ }^{*} \underline{\underline{B_{e}}} d \Omega+2 \int_{\Omega} \underline{\underline{B_{e}}} \quad: \underline{\underline{B}} \underline{\underline{\Omega}} d \Omega \\
& -2 \int_{\Omega} \underline{\underline{B_{e}}} \stackrel{\underline{\underline{B_{e}}}}{\underline{\underline{D}}} d \Omega+\int_{\Omega} \underline{\underline{B_{e}}}{ }^{*}: \frac{G}{\eta} \underline{\underline{B_{e}}} \underline{\underline{\hat{B}_{e}}} d \Omega=0
\end{aligned}
$$

where the superscript $*$ denotes test quantities and $\underline{F}^{d}$ denotes the prescribed traction field over the boundary $\partial \Omega_{F}$ where the loads are imposed.

This strongly nonlinear problem (finite elastic displacements, elastic left Cauchy Green tensor $B$, non constant shear modulus $G$ and viscosity $\eta$ ), is solved using a classical Newton-Raphson iterative procedure. A consistent linearization is achieved with the Gâteaux operator (see Appendix).

The domain $\Omega$ is approximated by a set of 8 -nodes isoparametric rectangular elements. In the case of the classical incompressible problem (a mixed velocity and pressure formulation), the finite element methods are not stable, some of them showing spurious pressure oscillations if velocity and pressure spaces are not chosen carefully. To be stable, a mixed formulation must verify consistency. The well-known inf-sup condition or the LBB condition [5] guaranties the stability of a finite element velocity - pressure calculation. For our non classical four-fields approach $\left(\underline{V}, p, q\right.$ and $\left.\underline{\underline{B}}_{\mathrm{e}}\right)$, we tested different interpolations to choose the accurate solution. We solved our problem considering different shape functions and examined the convergence in regrads of the element size. The error is calculated between numerical and analytical results of stress in the isothermal equi-biaxial elongation case. We obtain the best choice interpolation when velocity $V$ and the elastic $\underline{\underline{B}}$ e tensor are quadratic and for $p$ and $q$ linear.

The numerical simulation of the stretch-blow moulding process with the proposed visco-hyperelastic model consists in solving the set of thermo-mechanical equations on section $\Omega$ of 
a preform. The mechanical part is written in Eq. 13 and the thermal part is:

$$
\left\{\begin{array}{l}
\rho C_{p} \int_{\Omega} T^{*} \dot{T} d \Omega+k \int_{\Omega} \underline{\nabla} T^{*} \underline{\nabla} T d \Omega \\
=\int_{\Omega} T^{*}(\underline{\underline{\sigma}}: \underline{\underline{D}}) d \Omega-h \int_{\partial \Omega_{q}} T^{*}\left(T-T_{\infty}\right) d S \\
T=T_{0} \quad \text { at } \quad t=0
\end{array}\right.
$$

The conductivity term is introduced in this numerical solution.

\section{SIMULATION OF THE THERMO-MECHANICAL BIAXIAL PROBLEM}

As the self heating effect is not neglectible and produces an important effect on the viscous part of the model, the first estimation of the parameters obtained from our isothermal identification must be adjusted. An optimisation procedure in the Matlab Optimization Toolbox was chosen: the 'fminunc' function was used for this unconstrained nonlinear optimization. This function chooses the large-scale algorithm which is a subspace trust region method and is based on the interiorreflective Newton method described in $[8,9]$. Each iteration involves the approximate solution of a large linear system using the preconditioned conjugate gradients method. Almost a hundred simulations must be achieved to obtain the adjustment wanted. Finally, the characteristics of the PET for these viscohyperelastic model expressions to represent conveniently the biaxial experimental tension tests [4] are listed in the table 2 .

TABLE 2. THE CHARACTERISTICS OF THE PET

\begin{tabular}{|c|c|c|c|}
\hline \multirow{2}{*}{$\mathbf{G}\left(\boldsymbol{\varepsilon}_{\mathrm{e}}\right)$} & \multicolumn{2}{|c|}{$\mathrm{G}_{0}$} & \multirow{2}{*}{$\frac{8 \mathrm{MPa}}{0.001}$} \\
\hline & $\Lambda$ & & \\
\hline \multirow{12}{*}{$\mathbf{H}\left(\boldsymbol{\varepsilon}_{\mathrm{v}}, \overline{\dot{\varepsilon}}_{v}, \mathbf{T}\right)$} & \multirow{3}{*}{$f\left(\overline{\dot{\varepsilon}_{v}}\right)$} & $\lambda$ & 9.91 \\
\hline & & $\mathrm{a}$ & 2 \\
\hline & & $\mathrm{m}$ & 0.2 \\
\hline & \multirow{5}{*}{$h\left(\overline{\varepsilon_{v}}\right)$} & $\eta_{0}$ & 8.4 MPa.s \\
\hline & & $\mathrm{K}$ & 3.2 \\
\hline & & $\mathrm{h}_{0}$ & -0.21 \\
\hline & & $\mathrm{N}$ & 0.42 \\
\hline & & $\varepsilon_{\text {vlim_ref }}$ & 1.83 \\
\hline & \multirow{2}{*}{$\eta_{0}(\mathrm{~T})$} & $\mathrm{C}_{1}$ & 1.88 \\
\hline & & $\mathrm{C}_{2}$ & $25.81^{\circ} \mathrm{C}$ \\
\hline & \multirow{2}{*}{$\varepsilon_{\mathrm{vlim}}(\mathrm{T})$} & $\mathrm{B}_{1}$ & 0.07 \\
\hline & & $\mathrm{B}_{2}$ & $111.88^{\circ} \mathrm{C}$ \\
\hline
\end{tabular}

With the identified parameters, we can obtain the stressstrain curves from the thermo-visco-hyperelastic model. The stresses and the temperature are slightly heterogeneous in the specimen. For example, under a $8 \mathrm{~s}^{-1}$ strain rate, when the nominal strain rate reaches 1.8 , the final radial stress field $\left(\sigma_{\mathrm{rr}}\right)$ and the temperature field are shown on figure 4. One can see that stress varies from 14.2 $\mathrm{MPa}$ to $14.6 \mathrm{MPa}$ (about 2.8\%) and that temperature varies from $96^{\circ} \mathrm{C}$ to $99^{\circ} \mathrm{C}$. The conductivity term was took into account in the numerical simulation. There is about $3^{\circ} \mathrm{C}$ 's difference in the temperature field.
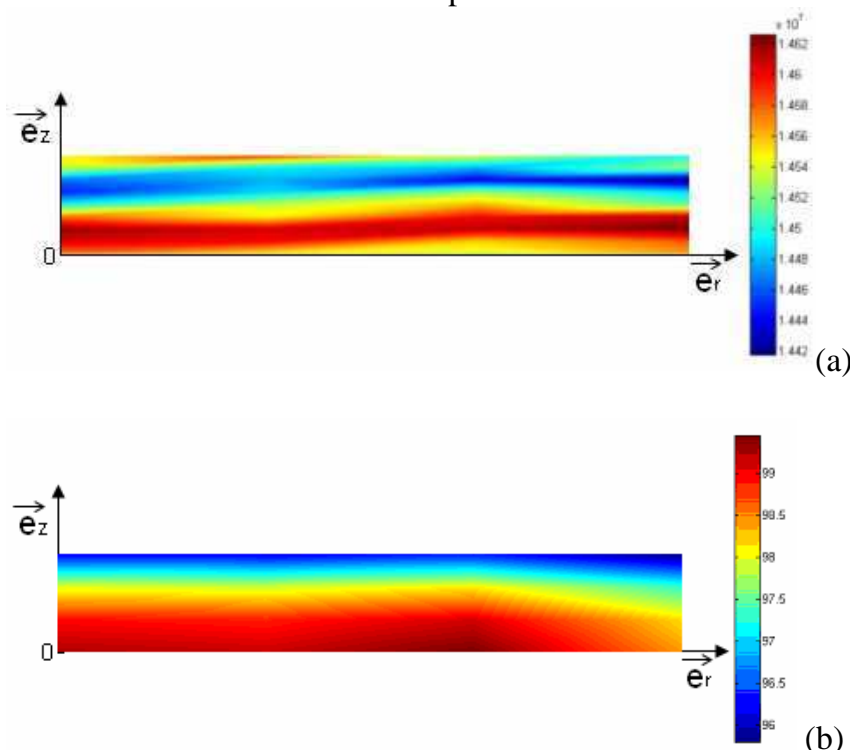

(b)

FIGURE 4. (a) The $\sigma_{\mathrm{rr}}$ stress field; (b) the temperature field at final stage

Figure 5 shows the comparison between the stresses obtained from this thermo-mechanical simulation and from the experimental data.

We listed the absolute errors between the thermomechanical simulation and the experimental data in the table 3 . The mean difference does not exceed $10 \%$ for each deformation rate.

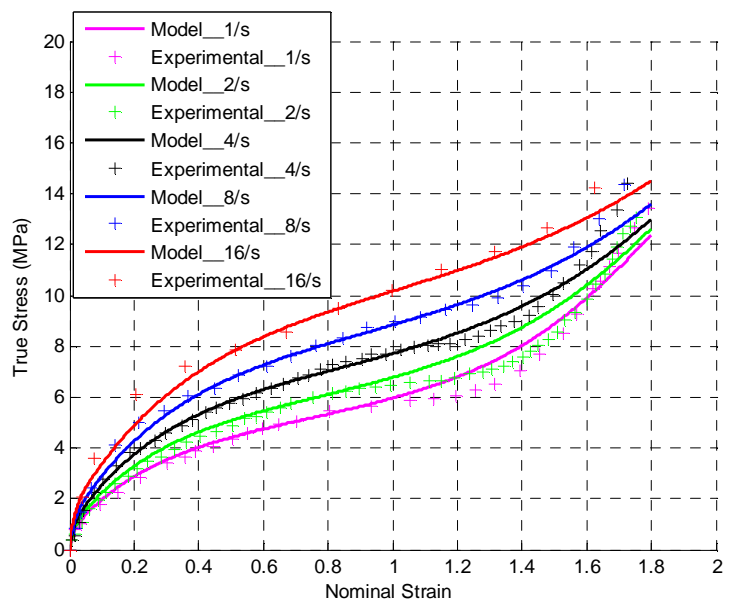

FIGURE 5. The experimental data (dots) and the thermomechanical results (lines) 
TABLE 3. ERRORS BETWEEN THE EXPERIMENTAL AND THE RESULTS OF THE MODEL

\begin{tabular}{c|c}
\hline Strain Rate (/s) & Absolute Error (\%) \\
\hline 1 & 5.52 \\
\hline 2 & 7.89 \\
\hline 4 & 5.83 \\
\hline 8 & 5.28 \\
\hline 16 & 5.75 \\
\hline
\end{tabular}

Evolutions of the temperature and the viscosity are shown in figure 6 . We focus on the temperature in the midline of the specimen thickness.

We can notice that the increasing evolution of the temperature versus strain is nearly linear. Furthermore, the self heating of the specimen for the strain rates from $1 \mathrm{~s}^{-1}$ to $16 \mathrm{~s}^{-1}$ are similar, but for the higher strain rate $32 \mathrm{~s}^{-1}$, the temperature increases more. It confirms that at very high deformation rate, the adiabatic heating due to the viscous dissipation, generates a significant temperature rises. The slope of the curve for the strain rate $32 \mathrm{~s}^{-1}$ is more important than the one for the other strains rates, therefore the strain hardening effect is not so considerable at $32 \mathrm{~s}^{-1}$. We can see in the figure $6 \mathrm{~b}$, the viscosity increases when the temperature rises.
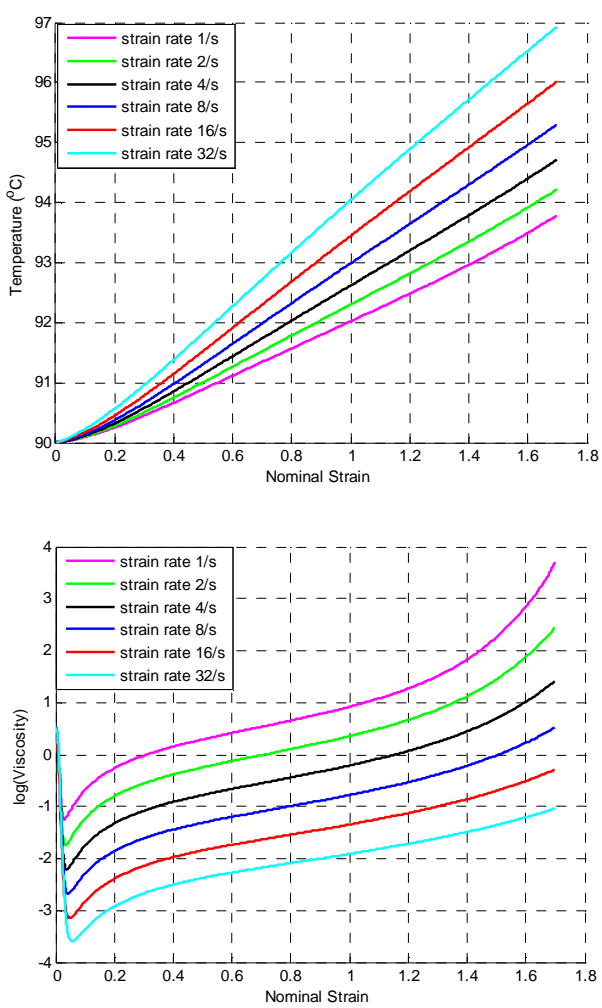

FIGURE 6. (a) The evolution of temperature under different strain rates; (b) The variation of viscosity with temperature under different strain rates.
If we examine the high strain rate case, for example, at $32 \mathrm{~s}^{-1}$ for $90^{\circ} \mathrm{C}$, the coupled themo-visco-hyperelastique model simulation gives the true stress $\sigma_{\text {rr }}$ evolution plotted on figure 7 which have only $7.83 \%$ errors with the experimental data. One can see that the stress evolution measured during the experiment saturates and seems to decrease when approaching the 1.8 elongation. This may be explained by the significantly higher temperature level coming from the self heating of the specimen for this test. Our model does not predict the decreasing shape but gives a pretty good estimation on the evolution with nearly no strain hardening effect for this case.

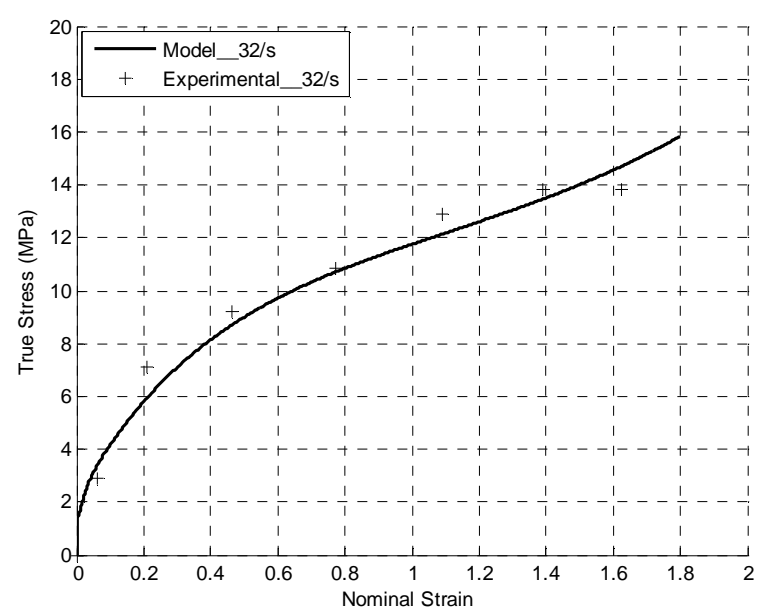

FIGURE 7. The experimental data (the points) and the thermo-mechanical results at $32 \mathrm{~s}^{-1}$ for $90^{\circ} \mathrm{C}$

\section{CONCLUSIONS}

Experiments have been conducted to characterise the thermal properties of the PET in the range of the ISBM temperature. Thermals imaging have been used to determine the surface temperature distribution of the PET sheets which are heated by infrared lamps. The Monte Carlo method is used to provide the parameters best fit from the temperature evolution.

The coupled thermo-visco-hyperelastic model proposed has been used to manage a finite element simulation of the equi biaxial elongation test. The weak form of the model has been implemented in Matlab. It shows that the self heating effect has an important effect on the viscous part of the model and that the identification of the parameters has to be adjusted by nonisothermal simulations.

The axi-symmetric version of the visco-hyperelastic model coupled to temperature is implemented and may be used in order to simulate the ISBM process. 


\section{ACKNOWLEDGEMENT}

This work could not possible without lamps IR given by Sidel Company and without injection of the PET sheets made by the LIM at Arts et Métiers ParisTech. Special thanks to G. Menary of QUB for his study on the experiments.

\section{REFERENCES}

[1] L. Figiel, C.P. Buckley. On the modeling of highly elastic flows of amorphous thermoplastic. International Journal of Non-linear Mechanics, 44, 389-395, (2009).

[2] Luo Y.M., Chevalier L., Monteiro E., Identification of a Visco-Elastic Model for PET Near Tg Based on Uni and Biaxial Results, The 14th International ESAFORM Conference on Material Forming, Queen's University , Belfast, Irlande du Nord, April 27-29, 2011.

[3] B. Cosson, L. Chevalier, J. Yvonnet. Optimization of the thickness of PET bottles during stretch blow molding by using a mesh-free (numerical) method. International Polymer Processing, Volume 24, Issue 3, Pages 223-233, (2009).

[4] G.H. Menary, C.W. Tan, E.M.A. Harkin-Jones, C.G. Armstrong, P.J. MartinBiaxial Deformation of PET at Conditions Applicable to the Stretch Blow Molding Process. Polymer Engineering \& Science, in press.

[5] Modest, M., Radiative Heat Transfer, Mc Graw-Hill,. New York, 1993.

[6] M. Bordival, Modélisation et optimisation numérique de l'étape de chauffage infrarouge pour la fabrication de bouteilles en PET par injection-soufflage, Thèse de doctorat, 2009.(in French)

[7] S. Monteix, F. Schmidt, Y. L. Maoult, R.B. Yedder, R.W. Diraddo, D. Laroche. Experimental study and numerical simulation of preform or sheet exposed to infrared radiative heating. J. Materials Processing Technology 119(1-3):90-97, (2001).

[8] F. Brezzi, M. Fortin, Mixed and hybrid Finite Element Methods, Springer :New York, 1991.

[9] Coleman, T.F. and Y. Li, "On the Convergence of Reflective Newton Methods for Large-Scale Nonlinear Minimization Subject to Bounds," Mathematical Programming, Vol. 67, Number 2, pp. 189-224, 1994.

\section{APPENDIX}

The four-fields approach is adopted for the numerical implementation. The weak form is written in the equation 13. since it's a strongly non linear problem, an iterative NewtonRaphson scheme is used. A consistent linearization is achieved with the help on the Gâteaux operator:

$$
\begin{aligned}
& D_{\Delta V}\left\{R_{1}\right\}=2 \eta_{n} \int_{\Omega} \underline{\underline{D}}^{*} \cdot \Delta \underline{\underline{D}} d \Omega \\
& D_{\Delta P}\left\{R_{1}\right\}=-\int_{\Omega} \underline{\underline{D}}^{*}: I P d \Omega \\
& \left\{D_{\Delta p}\left\{R_{1}\right\}=-\int_{\Omega} \underline{\underline{D}}^{*}: I p d \Omega\right. \\
& D_{\Delta B_{e}}\left\{R_{1}\right\}=\int_{\Omega} \underline{\underline{D}}^{*}: G_{\text {nonlin }} \Delta \underline{\underline{B_{e}}} d \Omega \\
& +\int_{\Omega} \underline{\underline{D^{*}}} \stackrel{\underline{B_{e}}}{D_{\Delta B}}\left\{G_{\text {nonlin }}\right\} d \Omega \\
& \left\{\begin{array}{l}
D_{\Delta V}\left\{R_{2}\right\}=-\int_{\Omega} P^{*} \underline{\underline{I}}: \Delta \underline{\underline{D}} d \Omega \\
D_{\Delta P}\left\{R_{2}\right\}=0 \\
D_{\Delta p}\left\{R_{2}\right\}=0 \\
D_{\Delta B_{e}}\left\{R_{2}\right\}=0
\end{array}\right. \\
& D_{\Delta V}\left\{R_{3}\right\}=0 \\
& D_{\Delta P}\left\{R_{3}\right\}=0 \\
& \left\{D_{\Delta p}\left\{R_{3}\right\}=0\right. \\
& D_{\Delta B_{e}}\left\{R_{3}\right\}=-\int_{\Omega} p^{*}\left(\operatorname{det} B_{e}-1\right) \underline{\underline{I}}: \underline{\underline{B_{e}}} d \Omega \\
& \left\{\begin{array}{l}
D_{\Delta V}\left\{R_{4}\right\}=2 \int_{\Omega} \underline{\underline{B_{e}}}: \underline{\underline{B_{e}}} \Delta \underline{\underline{\Omega}} d \Omega-2 \int_{\Omega} \underline{\underline{B_{e}^{*}}}: \underline{\underline{B_{e}}} \underline{\underline{D}} d \Omega \\
D_{\Delta P}\left\{R_{4}\right\}=0
\end{array}\right. \\
& D_{\Delta P}\left\{R_{4}\right\}=0 \\
& \left\{D_{\Delta B}\left\{R_{4}\right\}=\int_{\Omega} \underline{\underline{B_{e}}}{ }^{*} \cdot \underline{\underline{\dot{B}_{e}}} d \Omega-2 \int_{\Omega} \underline{\underline{B_{e}}} \stackrel{\underline{\underline{\Omega}}}{\underline{B_{e}}} d \Omega-2 \int_{\Omega} \underline{\underline{B_{e}}}{ }^{*}: \underline{\underline{D}} \Delta \underline{\underline{B_{e}}} d \Omega\right. \\
& +\int_{\Omega} \underline{\underline{B_{e}}}{ }^{*}: \frac{G_{\text {nonlin }}}{\eta_{\text {nonlin }}} \underline{\underline{B_{e}}} \Delta \underline{\underline{\hat{B}_{e}}} d \Omega+\int_{\Omega} \underline{\underline{B_{e}}}{ }^{*}: \frac{G_{\text {nonlin }}}{\eta_{\text {nonlin }}} \underline{\underline{\hat{B}_{e}}} \Delta \underline{\underline{B_{e}}} d \Omega \\
& +\int_{\Omega} \underline{\underline{B_{e}}}: \underline{\underline{B_{e}}} \underline{\underline{\hat{B}_{e}}} D_{\Delta B}\left\{\frac{G_{\text {nonlin }}}{\eta_{\text {nonlin }}}\right\} d \Omega
\end{aligned}
$$

Once the linearization has been done, we can implement it in the finite element code. In this work, we use a self made finite element code developed in the Matlab environment. The system to solve takes the following matrix form:

$\left.\left[\begin{array}{cccc}{\left[D_{\Delta V}\left\{R_{1}\right\}\right]} & {\left[D_{\Delta P}\left\{R_{1}\right\}\right]} & \left.\mid D_{\Delta p}\left\{R_{1}\right\}\right] & {\left[D_{\Delta B_{e}}\left\{R_{1}\right\}\right.} \\ {\left[D_{\Delta V}\left\{R_{2}\right\}\right]} & {[0]} & {[0]} & {[0]} \\ {\left[D_{\Delta V}\left\{R_{3}\right\}\right]} & {[0]} & {[0]} & {[0]} \\ {\left[D_{\Delta V}\left\{R_{4}\right\}\right]} & {[0]} & {[0]} & {\left[D_{\Delta B_{e}}\left\{R_{4}\right\}\right.}\end{array}\right]\right\}\left\{\begin{array}{l}{[\Delta V]} \\ {[\Delta P]} \\ {[\Delta p]} \\ {\left[\Delta B_{e}\right]}\end{array}\right\}=-\left\{\begin{array}{l}{\left[R_{1}\right]} \\ {\left[R_{2}\right]} \\ {\left[R_{3}\right]} \\ {\left[R_{4}\right]}\end{array}\right\}$

